TRANSACTIONS OF THE

AMERICAN MATHEMATICAL SOCIETY

Volume 358, Number 5, Pages 2071-2089

S 0002-9947(05)03716-5

Article electronically published on March 31, 2005

\title{
RESONANCES FOR STEPLIKE POTENTIALS: FORWARD AND INVERSE RESULTS
}

\author{
T. CHRISTIANSEN
}

\begin{abstract}
We consider resonances associated to the one dimensional Schrödinger operator $-\frac{d^{2}}{d x^{2}}+V(x)$, where $V(x)=V_{+}$if $x>x_{M}$ and $V(x)=V_{-}$if $x<-x_{M}$, with $V_{+} \neq V_{-}$. We obtain asymptotics of the resonance-counting function for several regions. Moreover, we show that in several situations, the resonances, $V_{+}$, and $V_{-}$determine $V$ uniquely up to translation.
\end{abstract}

\section{INTRODUCTION}

Suppose $V \in L^{\infty}(\mathbb{R} ; \mathbb{R})$ is "steplike" in the sense that there exist constants $x_{M}, V_{+}, V_{-}, V_{+} \neq V_{-}$, so that $V(x)=V_{ \pm}$when $\pm x>x_{M}$. We will study the resonances, or scattering poles, associated with the one dimensional Schrödinger operator $-\frac{d^{2}}{d x^{2}}+V$. The resolvent $R(z)=\left(-\frac{d^{2}}{d x^{2}}+V-z\right)^{-1}$ is bounded on $L^{2}(\mathbb{R})$ for all but a finite number of $z \in \mathbb{C} \backslash \mathbb{R}_{+}$. Considered as an operator from $L_{\text {comp }}^{2}(\mathbb{R})$ to $H_{\text {loc }}^{2}(\mathbb{R})$, it has a meromorphic continuation to the Riemann surface $\hat{Z}$ which is the minimal Riemann surface on which $\left(z-V_{+}\right)^{1 / 2}$ and $\left(z-V_{-}\right)^{1 / 2}$ are singlevalued holomorphic functions. This follows from the same techniques as used to study manifolds with cylindrical ends [7, 11, although this case is much simpler (see Section 3 for an explanation). In fact, one motivation for studying steplike potentials is the similarity their scattering theory has to the scattering theory for manifolds with cylindrical ends, and a hope that an understanding of the steplike case will shed some light on what can happen in the cylindrical ends case.

Choose a $\chi \in C_{c}^{\infty}(\mathbb{R})$ so that $\chi=1$ on the support of $V^{\prime}$. Then the poles of $\chi R(z) \chi$ are independent of the choice of $\chi$ with this property and we set

$$
\mathcal{R}=\left\{z_{j} \in \hat{Z}: \chi R(z) \chi \text { has a pole at } z_{j}\right\} .
$$

We list the elements with their multiplicities.

Let $\Pi: \hat{Z} \rightarrow \mathbb{C}$ be the natural projection operator and let $r_{ \pm}(z)=\left(z-V_{ \pm}\right)^{1 / 2}$. If $V_{+} \neq V_{-}, \hat{Z}$ is a four-fold cover of the plane. We identify the part of $\hat{Z}$ with $\operatorname{Im} r_{+}(z)>0$ and $\operatorname{Im} r_{-}(z)>0$ as the "physical sheet," that is, the sheet of $\hat{Z}$ on which $R(z)$ is a bounded operator on $L^{2}(\mathbb{R})$.

Poles of $R(z)$ are called resonances. They are in some sense analogous to eigenvalues and can, in many settings, be viewed as corresponding to decaying waves.

Received by the editors March 4, 2003 and, in revised form, March 31, 2004.

2000 Mathematics Subject Classification. Primary 34L25, 34A55, 81U40, 81 U05.

Key words and phrases. Steplike potentials, Schrödinger operator, resonances, inverse problem. This work was partially supported by NSF grant 0088922 .

(C)2005 American Mathematical Society Reverts to public domain 28 years from publication 
See [17, 20, for an introduction to resonances and a survey of some results on their distribution, or 21] for a less formal introduction.

Although resonances have been studied in many settings, there are relatively few cases where the asymptotics of the resonance counting function are known. Many of these are in some sense one dimensional or degenerate; cf. [5, 12, 13, 15, 16, 19. Our first theorem is

Theorem 1.1. Suppose $V_{+} \neq V_{-}$, and the convex hull of the support of $V^{\prime}$ is $[a, b]$. Then

$$
\#\left\{z_{j} \in \mathcal{R}:\left|\Pi\left(z_{j}\right)\right| \leq r^{2}, \operatorname{Im} r_{+}\left(z_{j}\right)<0, \text { and } \operatorname{Im} r_{-}\left(z_{j}\right)<0\right\}=\frac{2}{\pi}(b-a) r+o(r) .
$$

In case $V_{+}=V_{-}$, this theorem is due to [13, 19. Another proof for the $V_{+}=V_{-}$ case is given in [5], and additional results can be found in [14]. Our proof has more in common with the proof of [5], though we have to make adaptations due to the nature of $\hat{Z}$. In addition, rather than study the zeros of a function holomorphic on all of $\hat{Z}$, we study the zeros of functions meromorphic on $\hat{Z}$, in a region where they are holomorphic except at a finite number of points.

The next theorem follows by similar techniques, although we must make stronger assumptions on the perturbation.

Theorem 1.2. Suppose $V(x)=V_{+} H(x-\beta)+V_{-} H(\beta-x)+p(x)$, with $p \in C_{\text {comp }}^{0}(\mathbb{R})$ and $p^{\prime} \in L^{1}(\mathbb{R})$, and the convex hull of the support of $p$ is $\left[-b_{1}, b_{1}\right]$. Then, if $b_{1}>\beta$,

$$
\#\left\{z_{j} \in \mathcal{R}:\left|\Pi\left(z_{j}\right)\right| \leq r^{2}, \operatorname{Im} r_{+}\left(z_{j}\right)<0 \text { and } \operatorname{Im} r_{-}\left(z_{j}\right)>0\right\}=\frac{2\left(b_{1}-\beta\right)}{\pi} r+o(r)
$$

and, if $b_{1}>-\beta$,

$\#\left\{z_{j} \in \mathcal{R}:\left|\Pi\left(z_{j}\right)\right| \leq r^{2}, \operatorname{Im} r_{+}\left(z_{j}\right)>0\right.$ and $\left.\operatorname{Im} r_{-}\left(z_{j}\right)<0\right\}=\frac{2\left(\beta+b_{1}\right)}{\pi} r+o(r)$.

Here and elsewhere $H$ is the Heaviside function. If $\beta \in\left(-b_{1}, b_{1}\right)$, then we see that the distribution of poles on these sheets depends rather heavily on the value of $\beta$. It therefore seems unlikely that on one of these sheets (say, $\operatorname{Im} r_{+}>0, \operatorname{Im} r_{-}<0$ ) an asymptotic formula holds in general. However, we can prove the weaker

Theorem 1.3. Suppose $V_{+} \neq V_{-}$, and the convex hull of the support of $V^{\prime}$ is $[a, b]$. Then

$$
\begin{aligned}
& \#\left\{z_{j} \in \mathcal{R}:\left|\Pi\left(z_{j}\right)\right| \leq r^{2}, \operatorname{Im} r_{+}\left(z_{j}\right) \leq 0 \text { and } \operatorname{Im} r_{-}\left(z_{j}\right) \geq 0\right\} \\
& +\#\left\{z_{j} \in \mathcal{R}:\left|\Pi\left(z_{j}\right)\right| \leq r^{2}, \operatorname{Im} r_{+}\left(z_{j}\right)>0 \text { and } \operatorname{Im} r_{-}\left(z_{j}\right)<0\right\}=\frac{2}{\pi}(b-a) r+o(r) .
\end{aligned}
$$

Zworski 22 and Korotyaev 8 showed that resonances determine a compactly supported, even potential in one dimension if 0 is not a pole of the resolvent, and that there are two such potentials with the same resonances if 0 is a pole of the resolvent. The papers [1] and 9] have studied a similar question for compactly supported potentials on a half-line. Here we give an example of our inverse results for steplike potentials. Further results can be found in Section 5 .

Proposition 1.1. Suppose we know all the poles of $R(z), V_{+}, V_{-}$(with $V_{+} \neq$ $\left.V_{-}\right)$, and know that our potential $V$ has $V^{\prime}$ compactly supported. If, in addition, $\Pi^{-1}\left(V_{+}\right) \cap \mathcal{R} \neq \emptyset$, then $V$ is uniquely determined up to translation. 
It is clear that the qualifier "up to translation" cannot be removed, as $V(x)$ and $V(x-c), c \in \mathbb{R}$, have the same resonances. The techniques of the proof are similar to those Zworski [22] used in recovering an even, compactly supported potential on $\mathbb{R}$. This problem is more difficult because on $\hat{Z}$ we do not have the simple Weierstrass factorization that we have on $\mathbb{C}$. On the other hand, because $V_{+} \neq V_{-}$, some "symmetry" is broken and we have more identities at our disposal than when $V_{+}=V_{-}$. A related issue is that $\hat{Z}$ is "bigger" than $\mathbb{C}$, and thus there are "more" resonances and they can carry more information.

The techniques developed here for the forward problem are applied in 3 to obtain results for the distribution of resonances for potential scattering on cylinders.

We shall assume throughout that $V_{+}<V_{-}$and $V$ is real-valued.

\section{Preliminaries From COMPlex ANALYSis}

In this section we recall some results and language of complex analysis, e.g. [10, and prove a theorem we shall need on the distribution of zeros of functions which are "good" in a half-plane.

We shall often work with functions that are holomorphic not in the whole plane but are holomorphic within an angle $\left(\theta_{1}, \theta_{2}\right)$. A function $F$ holomorphic in an angle $\left(\theta_{1}, \theta_{2}\right)$ is of order $\rho$ there if

$$
\varlimsup_{r \rightarrow \infty} \frac{\ln \ln \left(\sup _{\theta \in\left(\theta_{1}, \theta_{2}\right)}\left|F\left(r e^{-\theta}\right)\right|\right)}{\ln r}=\rho .
$$

A function of order $\rho$ in the angle $\left(\theta_{1}, \theta_{2}\right)$ is of type $\tau$ there if

$$
\varlimsup_{r \rightarrow \infty} \frac{\ln \sup _{\theta \in\left(\theta_{1}, \theta_{2}\right)}\left|F\left(r e^{i \theta}\right)\right|}{r^{\rho}}=\tau .
$$

A function of order 1 and type $\tau<\infty$ (in an angle $\left(\theta_{1}, \theta_{2}\right)$ ) is said to be of exponential type there. Of course, $\rho$ and $\tau$ can depend on $\left(\theta_{1}, \theta_{2}\right)$.

The indicator of a function $F$ holomorphic in an angle $\theta_{1}<\arg z<\theta_{2}$ and of order $\rho$ is

$$
h_{F}(\theta)=\varlimsup_{r \rightarrow \infty} \frac{\ln \left|F\left(r e^{i \theta}\right)\right|}{r^{\rho}} .
$$

A function $F$ is of completely regular growth within the angle $\left(\theta_{1}, \theta_{2}\right)$ if

$$
\lim _{\substack{r \rightarrow \infty \\ r \notin E}} \frac{\ln \left|F\left(r e^{i \theta}\right)\right|}{r^{\rho}}=h_{F}(\theta),
$$

where the set $E \subset \mathbb{R}_{+}$is of zero relative measure and the convergence is uniform for $\theta \in\left(\theta_{1}, \theta_{2}\right)$.

We shall abuse notation slightly and also use the language above for a function that is holomorphic for $\theta_{1} \leq \arg z \leq \theta_{2}$ and $z$ outside of a compact set.

For a function $f$ defined in the upper half plane, define

$$
n_{f+}(r)=\#\left\{z_{j}: \operatorname{Im} z_{j}>0 \text { and } f\left(z_{j}\right)=0,\left|z_{j}\right|<r\right\} .
$$

Here, as elsewhere, all zeros are counted with multiplicities.

Theorem 2.1. Suppose $f(z)$ is holomorphic in the closed upper half plane $\operatorname{Im} z \geq 0$,

$$
|f(z)| \leq C e^{C|z|} \quad \text { for } \operatorname{Im} z \geq 0,
$$


$f(0)=1$,

$$
\left|\int_{-\infty}^{\infty} \frac{d[\arg f(t)]}{d t} d t\right|<\infty
$$

and

$$
\left|\int_{-\infty}^{\infty} \frac{\ln |f(t)|}{1+t^{2}} d t\right|<\infty
$$

Then

$$
\lim _{r \rightarrow \infty} \frac{n_{f+}(r)}{r}=\frac{1}{2 \pi} \int_{0}^{\pi} h_{f}(\varphi) d \varphi .
$$

A related result is Lemma 3.2 of 6 .

Proof. We first adopt some arguments of [10, Chapter III, Section 2]; a similar argument is used to prove Lemma 3.2 of [6]. By the principle of the argument,

$$
2 \pi n_{f+}(r)=\int_{-r}^{r} \frac{d[\arg f(t)]}{d t} d t+\int_{0}^{\pi} \frac{d\left[\arg f\left(r e^{i \varphi}\right)\right]}{r d \varphi} r d \varphi .
$$

We use the Cauchy-Riemann equations to rewrite this as

$$
2 \pi n_{f+}(r)=\int_{-r}^{r} \frac{d[\arg f(t)]}{d t} d t+r \int_{0}^{\pi} \frac{d}{d t}\left\{\ln \left|f\left(t e^{i \varphi}\right)\right|\right\}_{\mid t=r} d \varphi .
$$

Dividing both sides of the equation by $2 \pi r$, and integrating from 0 to $r$ results in

$$
\int_{0}^{r} \frac{n_{f+}(\rho)}{\rho} d \rho=\frac{1}{2 \pi} \int_{0}^{r} \frac{1}{\rho} \int_{-\rho}^{\rho} \frac{d[\arg f(t)]}{d t} d t d \rho+\frac{1}{2 \pi} \int_{0}^{\pi} \ln \left|f\left(r e^{i \varphi}\right)\right| d \varphi .
$$

By (2),

$$
\left|\frac{1}{2 \pi} \int_{0}^{r} \frac{1}{\rho} \int_{-\rho}^{\rho} \frac{d[\arg f(t)]}{d t} d t d \rho\right| \leq C \ln r
$$

so that we can, given $\epsilon>0$, choose $r_{\epsilon}$ so that

$$
\left|\frac{1}{r} \int_{0}^{r} \frac{1}{\rho} \int_{-\rho}^{\rho} \frac{d[\arg f(t)]}{d t} d t d \rho\right| \leq \frac{\epsilon}{6}
$$

for all $r>r_{\epsilon}$.

Now we roughly follow the proof of [10, Theorem 3, Chapter III, Section 3]. By [10. Theorem 6, Chapter V, Section 4], (11) and (2) imply that $f(z)$ is a function of class A and completely regular growth for $0<\arg z<\pi$, and $h_{f}(\theta)=k \sin \theta$. Because $f$ is of completely regular growth, if $r$ does not belong to the exceptional set $E$ for the function $f$, then for a sufficiently large $r_{\epsilon}$ and all $r>r_{\epsilon}$ we have

$$
\left|\int_{0}^{\pi} \frac{\ln \left|f\left(r e^{i \varphi}\right)\right|}{r} d \varphi-\int_{0}^{\pi} h_{f}(\varphi) d \varphi\right|<\epsilon / 6 .
$$

Then we have, for $r>r_{\epsilon}$ not in the exceptional set,

$$
\left|\frac{1}{r} \int_{0}^{r} \frac{n_{f+}(\rho)}{\rho} d \rho-\frac{1}{2 \pi} \int_{0}^{\pi} h_{f}(\varphi) d \varphi\right|<\epsilon / 3 .
$$

Then

$$
\lim _{r \rightarrow \infty, r \notin E} \frac{1}{r} \int_{0}^{r} \frac{n_{f+}(\rho)}{\rho} d \rho=\frac{1}{2 \pi} \int_{0}^{\pi} h_{f}(\varphi) d \varphi .
$$

In the same manner as [10, proof of Theorem 3, Chapter III, we can show that the limit holds without the restriction that $r \notin E$. 
Again following [10], we have by the monotonicity of $n_{f+}(r)$ that for $k>1$,

$$
\frac{1}{r} n_{f+}(r) \ln k \leq \frac{1}{r} \int_{r}^{k r} \frac{n_{f+}(t)}{t} d t=\frac{1}{r} \int_{0}^{k r} \frac{n_{f+}(t)}{t} d t-\frac{1}{r} \int_{0}^{r} \frac{n_{f+}(t)}{t} d t,
$$

giving us

$$
\lim \sup _{r \rightarrow \infty} \frac{n_{f+}(r)}{r} \leq \frac{k-1}{\ln k} \frac{1}{2 \pi} \int_{0}^{\pi} h_{f}(\varphi) d \varphi
$$

for any $k>1$. Taking the limit as $k \downarrow 1$ we obtain

$$
\limsup _{r \rightarrow \infty} \frac{n_{f+}(r)}{r} \leq \frac{1}{2 \pi} \int_{0}^{\pi} h_{f}(\varphi) d \varphi .
$$

By a similar argument (taking $0<k<1$ ), we obtain

$$
\liminf _{r \rightarrow \infty} \frac{n_{f+}(r)}{r} \geq \frac{1}{2 \pi} \int_{0}^{\pi} h_{f}(\varphi) d \varphi .
$$

This proves the theorem.

We shall use another theorem whose proof is very similar to the previous one. If $f(z)$ is holomorphic in $\left\{z \in \mathbb{C}:|z| \geq R_{1}\right\}$, let

$$
n_{f, R_{1}}(r)=\#\left\{z_{j}: f\left(z_{j}\right)=0, R_{1}<\left|z_{j}\right| \leq r\right\} .
$$

Again, we count the zeros with multiplicities.

Theorem 2.2. For $|z| \geq R_{1}$, suppose $f(z)$ is holomorphic, $|f(z)| \leq C e^{C|z|}$, and $f$ is of completely regular growth. Then

$$
\lim _{r \rightarrow \infty} \frac{n_{f, R_{1}}(r)}{r}=\frac{1}{2 \pi} \int_{0}^{2 \pi} h_{f}(\varphi) d \varphi .
$$

Proof. As in the proof of the previous theorem, the principle of the argument followed by an application of the Cauchy-Riemann equations gives us, for $r>R_{1}$,

$$
2 \pi n_{f, R_{1}}(r)=r \int_{0}^{2 \pi} \frac{d}{d t}\left\{\ln \left|f\left(t e^{i \varphi}\right)\right|\right\}_{\mid t=r} d \varphi-R_{1} \int_{0}^{2 \pi} \frac{d}{d t}\left\{\ln \left|f\left(t e^{i \varphi}\right)\right|\right\}_{\mid t=R_{1}} d \varphi .
$$

Dividing both sides of the equation by $2 \pi r$ and integrating from $R_{1}$ to $r$ results in

$$
\begin{aligned}
\int_{R_{1}}^{r} \frac{n_{f, R_{1}}(\rho)}{\rho} d \rho=\frac{1}{2 \pi} \int_{0}^{2 \pi} \ln \mid & f\left(r e^{i \varphi}\right)\left|d \varphi-\frac{1}{2 \pi} \int_{0}^{2 \pi} \ln \right| f\left(R_{1} e^{i \varphi}\right) \mid d \varphi \\
& -\frac{1}{2 \pi} R_{1} \log \left(r / R_{1}\right) \int_{0}^{2 \pi} \frac{d}{d t}\left\{\ln \left|f\left(t e^{i \varphi}\right)\right|\right\}_{\mid t=R_{1}} d \varphi .
\end{aligned}
$$

Then the proof follows the proof of the previous theorem.

$$
\text { 3. Elementary SCATtering TheORY OF }-\frac{d^{2}}{d x^{2}}+V
$$

For $z \in \hat{Z}$, let $r_{ \pm}(z)=\left(z-V_{ \pm}\right)^{1 / 2}$. To a point $z \in \hat{Z}$ we may associate the two roots $r_{ \pm}(z)$, and (if we are consistent) we can determine a mapping $\hat{Z} \rightarrow \hat{Z}$ by its action on $r_{+}(z)$ and $r_{-}(z)$. We define three maps $w_{+}, w_{-}$, and $w_{+-}: \hat{Z} \rightarrow \hat{Z}$, by

$$
\begin{aligned}
r_{ \pm}\left(w_{ \pm}(z)\right) & =-r_{ \pm}(z), \\
r_{\mp}\left(w_{ \pm}(z)\right) & =r_{\mp}(z), \\
r_{ \pm}\left(w_{+-}(z)\right) & =-r_{ \pm}(z) .
\end{aligned}
$$

We shall return to these mappings shortly. 
We define two functions $\phi_{ \pm}(x, z)$ with the following properties:

$$
\begin{gathered}
\left(-\frac{d^{2}}{d x^{2}}+V(x)-z\right) \phi_{ \pm}(x, z)=0, \\
\phi_{+}(x, z)= \begin{cases}T_{-}(z) e^{-i r_{-}(z) x} & \text { when } x \rightarrow-\infty, \\
e^{-i r_{+}(z) x}+R_{-}(z) e^{i r_{+}(z) x} & \text { when } x \rightarrow \infty\end{cases}
\end{gathered}
$$

and

$$
\phi_{-}(x, z)= \begin{cases}e^{i r_{-}(z) x}+R_{+}(z) e^{-i r_{-}(z) x} & \text { when } x \rightarrow-\infty \\ T_{+}(z) e^{i r_{+}(z) x} & \text { when } x \rightarrow \infty\end{cases}
$$

Recall that we assume that $V_{+}<V_{-}$. The following identities are well known when $\Pi(z) \in \mathbb{R}$ (e.g. [4, Theorem 2.1]) and can be extended to $\hat{Z}$ using the meromorphy of $R_{ \pm}, T_{ \pm}$. We have translated them to statements on $\hat{Z}$ using the mappings $w_{ \pm}, w_{+-}$defined above to obtain

$$
\begin{aligned}
r_{-}(z) T_{-}(z) & =r_{+}(z) T_{+}(z), \\
R_{-}\left(w_{+}(z)\right) R_{-}(z) & =1 \\
T_{ \pm}(z) R_{ \pm}\left(w_{\mp}(z)\right) & =T_{ \pm}\left(w_{\mp}(z)\right), \\
-r_{-}(z) T_{-}(z) R_{+}\left(w_{+-}(z)\right) & =r_{+}(z) R_{-}(z) T_{+}\left(w_{+-}(z)\right), \\
T_{-}\left(w_{+-}(z)\right) T_{+}(z)+R_{-}\left(w_{+-}(z)\right) R_{-}(z) & =1 \\
T_{-}\left(w_{+-}(z)\right) T_{+}(z)+R_{+}\left(w_{+-}(z)\right) R_{+}(z) & =1 \\
R_{+}\left(w_{-}(z)\right) R_{+}(z) & =1 \\
-r_{-}(z) T_{-}(z) T_{-}\left(w_{-}(z)\right) & =-r_{+}(z) R_{-}\left(w_{-}(z)\right)+r_{+}(z) R_{-}(z), \\
r_{+}(z) T_{+}(z) T_{+}\left(w_{+}(z)\right) & =r_{-}(z) R_{+}\left(w_{+}(z)\right)-r_{-}(z) R_{+}(z) .
\end{aligned}
$$

We shall also want a family of reference operators, which we define below. For $\beta \in \mathbb{R}$, define the potential $V_{\beta}$ to be

$$
V_{\beta}(x)= \begin{cases}V_{-} & \text {if } x<\beta, \\ V_{+} & \text {if } x>\beta\end{cases}
$$

We define the corresponding set of generalized eigenfunctions, $\phi_{ \pm, \beta}(x, z)$, which in this case can be given explicitly:

$$
\phi_{+, \beta}(x, z)= \begin{cases}\frac{2 r_{+}(z)}{r_{+}(z)+r_{-}(z)} e^{i \beta\left(r_{-}(z)-r_{+}(z)\right)} e^{-i r_{-}(z) x} & \text { if } x<\beta, \\ e^{-i r_{+}(z) x}+\frac{r_{+}(z)-r_{-}(z)}{r_{+}(z)+r_{-}(z)} e^{-2 i r_{+}(z) \beta} e^{i r_{+}(z) x} & \text { if } x>\beta\end{cases}
$$

and

$$
\phi_{-, \beta}(x, z)= \begin{cases}e^{i r_{-}(z) x}+\frac{r_{-}(z)-r_{+}(z)}{r_{-}(z)+r_{+}(z)} e^{2 i r_{-}(z) \beta} e^{-i r_{-}(z) x} & \text { if } x<\beta, \\ \frac{2 r_{-}(z)}{r_{+}(z)+r_{-}(z)} e^{i \beta\left(r_{-}(z)-r_{+}(z)\right)} e^{i r_{+}(z) x} & \text { if } x>\beta .\end{cases}
$$

We shall denote

$$
R_{\beta}(z)=\left(-\frac{d^{2}}{d x^{2}}+V_{\beta}-z\right)^{-1} .
$$

From the explicit representation of $\phi_{ \pm, \beta}$ and the corresponding expression for the Schwartz kernel of $R_{\beta}(z)$ (cf. (19)), it is relatively easy to see that $R_{\beta}(z)$ : $L_{\text {comp }}^{2}(\mathbb{R}) \rightarrow H_{\text {loc }}^{2}(\mathbb{R})$ has a meromorphic continuation to $\hat{Z}$. Then, since $R(z)=$ $R_{\beta}(z)\left(I+\left(V-V_{\beta}\right) R_{\beta}(z)\right)^{-1}, R(z)$ also has a meromorphic continuation to $\hat{Z}$. 


\section{Asymptotics For THE NUMBer OF RESONANCES}

In this section we use knowledge about the relationship between poles of the resolvent and poles of $R_{ \pm}, T_{ \pm}$to obtain results on the asymptotics for the number of poles. Many or all of these results could be obtained by more closely following the methods of [5]. However, we shall want some of the intermediate results we obtain here for the inverse results of Section 5 .

To bound the number of resonances, we shall use the following proposition. It is essentially a restating of Proposition 3.3 of [2] for this special case. We remark that while the proofs of [2] are given for the Laplacian, or Laplacian plus compactly supported potential, on a manifold with cylindrical ends, they work almost without change for our setting.

Proposition 4.1. Let $z \in \hat{Z}$, and suppose $\Pi(z)$ is not in the point spectrum of $\frac{-d^{2}}{d x^{2}}+V$. Then, if $\operatorname{Im} r_{ \pm}(z)<0$ but $\operatorname{Im} r_{\mp}(z)>0$, then $z$ is a pole of $\chi R \chi$ if and only if $z$ is a pole of $R_{\mp}$, and the multiplicities coincide. If $\operatorname{Im} r_{-}(z)<0$ and $\operatorname{Im} r_{+}(z)<0$, then $z$ is a pole of $\chi R \chi$ if and only if it is a pole of $R_{-} R_{+}-T_{-} T_{+}$, and the multiplicities coincide.

In fact, in this simple case we can say more. Looking at the expansions for $\phi_{+}$at infinity, if $R_{-}$has a pole of order $k$ at $z_{0}$, then so does $T_{-}$. Then using (5), unless $\Pi\left(z_{0}\right)$ is $V_{+}$or $V_{-}, T_{+}$has a pole of the same order, and thus so does $R_{+}$. Suppose $\operatorname{Im} r_{-}(z)<0, \operatorname{Im} r_{+}(z)<0$, and $\Pi(z) \notin \operatorname{spec}\left(-\frac{d^{2}}{d x^{2}}+V\right)$. We note that

$$
\begin{gathered}
\left(R_{-} R_{+}(z)-T_{+} T_{-}(z)\right)^{-1}=\left(R_{-} R_{+}\right)\left(w_{+-}(z)\right)-\left(T_{+} T_{-}\right)\left(w_{+-}(z)\right), \\
T_{-}(z)=\frac{T_{-}\left(w_{+-}(z)\right)}{\left(R_{-} R_{+}-T_{+} T_{-}\right)\left(w_{+-}(z)\right)},
\end{gathered}
$$

and $T_{-}\left(z^{\prime}\right)$ is nonzero if $\Pi\left(z^{\prime}\right) \neq V_{+}, V_{-}$. Since $T_{ \pm}, R_{ \pm}$are regular at $w_{+-}(z)$, the multiplicity of $z$ as a pole of $R_{-} R_{+}-T_{-} T_{+}$is equal to its multiplicity as a pole of $T_{-}$, and thus of $R_{+}$.

We recall some results and notation of [2] that will be helpful in extending Proposition 4.1. Although the following lemma may be well known, one reference is Lemma 3.1 of [2].

Lemma. Suppose $A(z)$ is a $d \times d$-dimensional meromorphic matrix, invertible for some value of $z$. Then, near $z_{0}$, it can be put into the form

$$
A(z)=E(z)\left(\sum_{j=1}^{p}\left(z-z_{0}\right)^{-k_{j}} P_{j}+\sum_{j=p+1}^{p^{\prime}}\left(z-z_{0}\right)^{l_{j}} P_{j}+P_{0}\right) F(z),
$$

where $E(z), F(z)$, and their inverses are holomorphic near $z_{0}, k_{j}, l_{j} \in \mathbb{N}$ and $P_{i} P_{j}=\delta_{i j} P_{i}, \operatorname{tr} P_{0}=d-p^{\prime}, \operatorname{tr} P_{i}=1, i=1, \ldots, p^{\prime}$. The $k_{j}$ and $l_{j}$ are, up to rearrangement, uniquely determined.

Using the notation of the lemma, the "maximal multiplicity" $\mu m$ of $A$ at $z_{0}$ is

$$
\mu m_{z_{0}}(A)=\sum_{j=1}^{p} k_{j}
$$

(cf. [2, Definition 3.2]). 
The following proposition improves on Proposition 4.1.

Proposition 4.2. Let $z \in \hat{Z}$. If $\Pi(z) \neq V_{+}, V_{-}$, then the multiplicity of $z$ as a pole of the cut-off resolvent $\chi R \chi$ is equal to the multiplicity of $z$ as a pole of $T_{-}$, $T_{+}, R_{-}$, or $R_{+}$. If $\Pi(z)=V_{+}$, then $z$ is a pole of $\chi R \chi$ of order at most one and is a pole of $\chi R \chi$ if and only if $T_{-}(z) \neq 0$. If $\Pi(z) \in\left(V_{+}, V_{-}\right]$, then $z$ is not a pole of $\chi R \chi$ or of $T_{-}, T_{+}, R_{-}$or $R_{+}$.

Proof. By Proposition 4.1 and the subsequent remarks, we need only consider what happens when $\Pi(z)$ lies in the spectrum of $-\frac{d^{2}}{d x^{2}}+V$. We will use

$$
\phi_{ \pm}(x, z(k))=\phi_{ \pm, \beta}(x, z(k))-\left(R(z(k))\left(V-V_{\beta}\right) \phi_{ \pm, \beta}(\cdot, z(k))\right)(x) .
$$

Thus $R_{ \pm}, T_{ \pm}$cannot have a pole of multiplicity greater than the multiplicity of the pole of $R$.

First, suppose $z$ corresponds to an eigenvalue; that is, $z$ lies in the physical sheet and $\Pi(z)$ lies in the point spectrum of $-\frac{d^{2}}{d x^{2}}+V$. Then $\chi R \chi, T_{ \pm}, R_{ \pm}$all have poles of order one at $z$. Then $T_{ \pm}, R_{ \pm}$are regular at $w_{+}(z), w_{-}(z)$, and $w_{+-}(z)$. By [2, Theorem 3.1], then $\chi R \chi$ is regular at $w_{+}(z), w_{-}(z)$, and $w_{+-}(z)$.

By [11, Proposition 6.7] and the fact that there are no eigenvalues embedded in the continuous spectrum, if $z \in \mathcal{R}$ lies on the boundary of the physical sheet and $\Pi(z) \in\left[V_{+}, \infty\right)$, then $\Pi(z)=V_{+}$or $\Pi(z)=V_{-}$. Moreover, by Lemma 6.9 and Proposition 6.7 of [11, if $\Pi(z)=V_{-}, z$ is not a pole of the resolvent, so the entries in the scattering matrix must be regular there.

Because $R_{-}(z) R_{-}\left(w_{+}(z)\right)=1$ and $R_{-}\left(w_{+}(z)\right)=\bar{R}_{-}(z)$ when $\pi(z) \in\left[V_{+}, V_{-}\right]$, $R_{-}(z)$, and hence $T_{ \pm}(z), R_{+}(z)$ have no poles when $\Pi(z) \in\left(V_{+}, V_{-}\right)$. Then, by [11, Proposition 6.7] and [2, Theorem 3.1], $\chi R \chi$ has no poles in this region.

Next suppose that $\Pi(z) \in\left(V_{-}, \infty\right)$ but that $z$ does not lie on the boundary of the physical sheet. Let

$$
S_{2}(z)=\left(\begin{array}{cc}
R_{-}(z) & T_{+}(z) \\
T_{-}(z) & R_{+}(z)
\end{array}\right)
$$

By Theorem 3.1 of [2], the multiplicity of $z$ as a pole of $R$ is equal to its "maximal multiplicity" as a pole of $S_{2}$. Since, for the $z$ we are considering, $\left(S_{2}(z)\right)^{-1}=$ $S_{2}\left(w_{+-}(z)\right)=\bar{S}_{2}(z)$, the determinant of $S_{2}$ is regular for these values of $z$. Therefore, since $S_{2}$ is a $2 \times 2$ matrix, the maximal multiplicity of $z$ as a pole of $S_{2}$ is equal to its multiplicity as a pole of $R_{+}$(or $T_{ \pm}$or $R_{-}$).

Finally, we consider what happens for $z_{0}$ such that $\Pi\left(z_{0}\right)=V_{+}$. If $z_{0}$ lies on the boundary of the physical sheet, by [11, Proposition 6.7], $z_{0}$ is a pole of order one of $\chi R \chi$ if and only if $T_{-}\left(z_{0}\right) \neq 0$. Notice in this case that $T_{+}$and $R_{+}$have a pole at $z_{0}$ but are regular at $w_{-}\left(z_{0}\right)$. If $T_{+}$is regular at $z_{0}, \chi R \chi$ is regular at $z_{0}$.

Now suppose $z_{0}$ does not lie on the boundary of the physical sheet and $\Pi\left(z_{0}\right)=$ $V_{+}$. Then we will use

$$
R(z)-R\left(w_{+-}(z)\right)=\frac{i}{2 r_{+}(z)} \phi_{+}(z) \otimes \phi_{+}\left(w_{+-}(z)\right)+\frac{i}{2 r_{-}(z)} \phi_{-}(z) \otimes \phi_{-}\left(w_{+-}(z)\right) .
$$

Here we use the notation $(f \otimes g) h(x)=f(x) \int g\left(x^{\prime}\right) h\left(x^{\prime}\right) d x^{\prime}$. Note that $\phi_{+}$is holomorphic at $z_{0}$ and $w_{+-}\left(z_{0}\right)$. Considering the expansion at infinity of the residue of the kernel of $R(z)$, we see that $R$ has a pole at $z_{0}$ only if $\phi_{-}$, and thus $T_{+}$, has a pole at $z_{0}$. Thus, using (17), $z_{0} \in \mathcal{R}$ if and only if $T_{+}$has a pole of order 1 at $z_{0}$. 
We shall now study the behaviour of $T_{ \pm}(z), R_{ \pm}(z)$ when $z$ lies on the closure of the physical sheet $\left(\operatorname{Im} r_{+}(z)>0, \operatorname{Im} r_{-}(z)>0\right)$. It will be more convenient to introduce the variable $k=r_{+}(z)$ on its closure. We write $z=z(k)=\left(k^{2}+V_{+}\right)^{1 / 2}$ in this region. When $k_{0} \in \mathbb{R}$, we understand $z\left(k_{0}\right)$ to be the element of $\hat{Z}$ obtained by taking the limit of $z(k)$ as $k \rightarrow k_{0}, \operatorname{Im} k>0$.

The following proposition is related to Lemma 3.3 and Corollary 3.4 of [5].

Proposition 4.3. Suppose that $V^{\prime}$ is compactly supported. Then, on the closure of the set with $\operatorname{Im} k>0$ and $\operatorname{Im} r_{-}(z(k))>0,\left|T_{ \pm}(z(k))-1\right| \leq C /|k|$.

Proof. We shall assume that $\operatorname{supp} V^{\prime} \subset\left[-b_{1}, b_{1}\right]$, and that $b_{1}>0$.

If $\chi \in C_{c}^{\infty}(\mathbb{R})$ is 1 on the support of $V-V_{\beta}$,

$$
R(z) \chi=R_{\beta}(z) \chi \sum_{0}^{\infty}(-1)^{j}\left(\left(V-V_{\beta}\right) R_{\beta}(z) \chi\right)^{j}
$$

when $z$ is on the closure of the physical sheet and $|z|$ is sufficiently large. We shall apply this identity and (17) with $\beta=0$. The operator $e^{ \pm i k x} R_{\beta}(z(k)) e^{\mp i k x}$ has Schwartz kernel given by

$$
\begin{aligned}
\frac{e^{ \pm i k\left(x-x^{\prime}\right)}}{W_{\beta}(z(k))}( & H\left(x-x^{\prime}\right) \phi_{-, \beta}(x, z(k)) \phi_{+, \beta}\left(x^{\prime}, z(k)\right) \\
& \left.+H\left(x^{\prime}-x\right) \phi_{-, \beta}\left(x^{\prime}, z(k)\right) \phi_{+, \beta}(x, z(k))\right),
\end{aligned}
$$

where

$$
W_{\beta}(z(k))=W\left[\phi_{-, \beta}(x, z(k)), \phi_{+, \beta}(x, z(k))\right]=\frac{-4 i k r_{-}(z(k))}{k+r_{-}(z(k))} e^{i \beta\left(r_{-}(z(k))-k\right)}
$$

is the Wronskian.

Let $\tilde{\chi} \in C_{c}^{\infty}(\mathbb{R})$ and note that when $z$ is in the closure of the physical sheet, $r_{+}(z)=r_{-}(z)+O\left(1 /|z|^{1 / 2}\right)$. Examining (14), (15), (19), and (20), we see that

$$
\left\|\tilde{\chi}(x) e^{ \pm i k\left(x-x^{\prime}\right)} R_{0}\left(x, x^{\prime}, z(k)\right) \tilde{\chi}\left(x^{\prime}\right)\right\|_{L^{\infty}\left(\mathbb{R}_{x} \times \mathbb{R}_{x^{\prime}}\right)} \leq \frac{C}{|k|}
$$

when $k$ is in the closed upper half plane and $z$ is in the closure of the physical sheet. (The constant $C$ depends on $\tilde{\chi}$, of course.) Therefore, in this same region

$$
\left\|\tilde{\chi} e^{ \pm i k x} R_{0}(z(k)) \tilde{\chi} e^{\mp i k x}\right\|_{L^{2}(\mathbb{R}) \rightarrow L^{2}(\mathbb{R})} \leq \frac{C}{|k|}
$$

and, when $|k|$ is sufficiently large,

$$
\left.\| \sum_{1}^{\infty}(-1)^{j}\left(e^{ \pm i k x}\left(V-V_{0}\right) R_{0}(z(k)) \chi\right) e^{\mp i k x}\right)^{j} \| \leq \frac{C}{|k|} .
$$

Let

$$
\begin{aligned}
g(x, k) & =\sum_{0}^{\infty}(-1)^{j}\left(\left(V-V_{0}\right) R_{0}(z(k)) \chi\right)^{j}\left(V-V_{0}\right) \phi_{+, 0} \\
& =e^{-i k x} \sum_{0}^{\infty}(-1)^{j}\left(e^{i k x}\left(V-V_{0}\right) R_{0}(z) \chi e^{-i k x}\right)^{j}\left(V-V_{0}\right)\left(e^{i k x} \phi_{+, 0}\right) .
\end{aligned}
$$

Then $g$ is supported in $\left[-b_{1}, b_{1}\right]$, and using (14) and (21) we have

$$
\left\|e^{i k \cdot} g(\cdot, k)\right\| \leq C .
$$


Using (17), (18), and the explicit expression for the Schwartz kernel of $R_{\beta}(z)$, we obtain

$$
\begin{aligned}
& T_{-}(z(k))-\frac{2 k}{k+r_{-}(z(k))} \\
&=\frac{-1}{W_{0}(z(k))} \frac{2 k}{k+r_{-}(z(k))} \int_{-b_{1}}^{0}\left(e^{i r_{-}(z(k)) x^{\prime}}+\frac{r_{-}(z(k))-k}{r_{-}(z(k))+k} e^{-i r_{-}(z(k))}\right) g\left(x^{\prime}, k\right) d x^{\prime} \\
&-\frac{1}{W_{0}(z(k))} \frac{2 k}{k+r_{-}(z(k))} \int_{0}^{b_{1}} \frac{2 r_{-}(z(k))}{k+r_{-}(z(k))} e^{i k x^{\prime}} g\left(x^{\prime}, k\right) d x^{\prime} .
\end{aligned}
$$

Thus $\left|T_{-}(z(k))-1\right| \leq \frac{C}{|k|}$ for $k$ in the closed upper half plane, and $z$ in the closure of the physical space.

The result for $T_{+}$follows from the relation (5).

We recall a slight modification of Lemma 4.1 of [5].

Lemma 4.1. Suppose $h \in L^{\infty}(\mathbb{R})$ has support contained in $[-1,1]$, but in no smaller interval. Suppose $f(x, k)$ is analytic for $k$ in the closed upper half plane, and for real $k$ we have $f(x, k) \in L^{2}([-1,1] d x, \mathbb{R} d k)$. Then $\int e^{ \pm i k x} h(x)(1-f(x, k)) d x$ has exponential type at least 1 for $k$ in the upper half plane.

We will use this lemma to prove

Proposition 4.4. Suppose that $\left[-b_{1}, b_{1}\right]$ is the convex hull of the support of $V^{\prime}$. Then $R_{ \pm}(z(k))$ are, for $\operatorname{Im} k>0$, functions of exponential type and completely regular growth. Moreover, $R_{ \pm}(z(k))$ is of type $2 b_{1}$ in this region. For $t \in \mathbb{R}$, fixed $\alpha \geq 0$, and $z(t+i \alpha)$ in the closure of the physical sheet, $R_{ \pm}(z(t+i \alpha))=O\left(|t|^{-1}\right)$.

Proof. The proof of this proposition resembles that of the previous one, though we must use Lemma 4.1 .

We give the proof for $R_{-}$, as the proof for $R_{+}$is similar. Using the functions $g$ and $W_{0}$ defined in (22) and (20), respectively, we have

$$
R_{-}(z(k))-\frac{k-r_{-}(z(k))}{k+r_{-}(z(k))}=\frac{-1}{W_{0}(z(k))} \frac{2 r_{-}(z(k))}{k+r_{-}(z(k))}\left(I_{1}+I_{2}+I_{3}\right),
$$

where

$$
\begin{aligned}
& I_{1}(k)=\int_{-b_{1}}^{0} \frac{2 k}{k+r_{-}(z(k))} e^{-i r_{-}(z(k)) x^{\prime}} g\left(x^{\prime}, k\right) d x^{\prime}, \\
& I_{2}(k)=\int_{0}^{b_{1}} e^{-i k x^{\prime}} g\left(x^{\prime}, k\right) d x^{\prime}, \\
& I_{3}(k)=\int_{0}^{b_{1}} \frac{k-r_{-}(z(k))}{k+r_{-}(z(k))} e^{i k x^{\prime}} g\left(x^{\prime}, k\right) d x^{\prime} .
\end{aligned}
$$

Using (23) $,\left|I_{1}(k)\right| \leq C,\left|I_{3}(k)\right| \leq C$ when $\operatorname{Im} k \geq 0$ and $|k|$ is sufficiently large. We rewrite

$$
\begin{aligned}
I_{2}(k)= & \int_{0}^{b_{1}} e^{-2 i k x^{\prime}}\left(V-V_{0}\right)\left(x^{\prime}\right)\left(1+f_{1}\left(x^{\prime}, k\right)\right) d x^{\prime} \\
& +\int_{0}^{b_{1}} e^{-i k x^{\prime}} \frac{k-r_{-}(z(k))}{k+r_{-}(z(k))} e^{i k x^{\prime}}\left(V-V_{0}\right)\left(x^{\prime}\right) d x^{\prime},
\end{aligned}
$$


where

$$
f_{1}\left(x^{\prime}, k\right)=\sum_{1}^{\infty}(-1)^{j}\left(e^{i k x^{\prime}} R_{0}(z(k))\left(V-V_{0}\right) e^{-i k x^{\prime}}\right)^{j} e^{i k x^{\prime}} \phi_{+, 0} .
$$

The second integral in (27) is clearly bounded. Using (21), the first integral is, for large $|k|$, bounded by $C e^{2 b_{1} \operatorname{Im} k}$. This shows that $\left|R_{-}(z(t+i \alpha))\right|=O\left(|t|^{-1}\right)$.

The function $f_{1}(x, k)$ may have poles at a finite number of points with $\operatorname{Im} k \geq$ 0 (and $\operatorname{Im} r_{-}(z(k))>0$ ). These poles correspond to eigenvalues of $-\frac{d^{2}}{d x^{2}}+V$. Using (21), for $t$ real, and for all but a finite number of $\alpha \geq 0, f_{1}\left(x^{\prime}, t+i \alpha\right) \in$ $L^{2}\left(\left[-b_{1}, b_{1}\right] d x^{\prime}, \mathbb{R} d t\right)$. Now, by a shift of variable (using $k-i \alpha$, for $\alpha$ chosen greater than the largest imaginary part of a pole of $R(z(k)))$ followed by a rescaling, we may apply Lemma 4.1 to see that $I_{2}(k)$, and thus $R_{-}(z(k))$, is of type $2 b_{1}$.

Proof of Theorem 1.1. We continue to use the variable $k=r_{+}(z)$, working in the closed upper half plane (with $\operatorname{Im} r_{-}(z(k)) \geq 0$ ).

Suppose $k_{1}, k_{2}, \ldots, k_{n}$ are the poles, listed with multiplicity, of $\phi(k)=$ $\left(T_{-} T_{+}-R_{-} R_{+}\right)(z(k))$ for $k$ in the closed upper half plane. Let

$$
p(k)=\prod_{j=1}^{n}\left(1-k / k_{j}\right)
$$

Then $\phi_{1}(k)=p(k) \phi(k) / p(-k)$ is a holomorphic function in $\operatorname{Im} k>0$, with a continuous extension to the closed half plane. Now let $\phi_{2}(k)=\phi_{1}(k) / \phi_{1}(0)$ if $\phi_{1}(0) \neq 0$ and $\phi_{2}(k)=\phi_{1}(k) m ! / \phi_{1}^{(m)}(0) k^{m}$ if $\phi_{1}^{(j)}(0)=0$ for $0 \leq j<m$ and $\phi_{1}^{(m)}(0) \neq 0$.

By Proposition 4.3 ,

$$
\left(T_{-} T_{+}-R_{-} R_{+}\right)(z(k))=1-R_{-} R_{+}(z(k))+O\left(|k|^{-1}\right) .
$$

Thus

$$
h_{\phi_{2}(k)}(\varphi)=h_{R_{-} R_{+}(z(k))}(\varphi)=4 b_{1} \sin \varphi,
$$

using Proposition 4.4 and the fact that $R_{-}(z(k)), R_{+}(z(k))$ are of completely regular growth in the upper half plane. Since $R_{ \pm}(z(t))=O\left(|t|^{-1}\right)$ for $t$ in $\mathbb{R}$, we have $\left|\int_{-\infty}^{\infty} \frac{d\left[\arg \phi_{2}(t)\right]}{d t} d t\right|<\infty$. Although $\phi_{2}(k)$ is not holomorphic in the closed upper half plane, it is holomorphic in the closed upper half plane except at the points $k= \pm\left(V_{-}-V_{+}\right)^{1 / 2}$. It is continuous at these points (in fact, a stronger statement can be made), and that is enough to guarantee that we may apply Theorem 2.1 to obtain Theorem 1.1 .

We next turn to proving Theorem 1.2, For this, we shall need to better understand the behaviour of $R_{ \pm}(z(k))$ when $k \in \mathbb{R}$. Recall that $\operatorname{Im}\left(r_{-}(z(k))\right)>0$ when $\operatorname{Im} k>0$, and thus the restriction on the sign of $r_{-}(k)$ ensures that we are on the boundary of the physical sheet.

Lemma 4.2. Suppose $V(x)=V_{\beta}+p(x)$, with $p \in C_{\text {comp }}^{0}(\mathbb{R})$, and $p^{\prime} \in L^{1}(\mathbb{R})$, and the convex hull of the support of $p$ is $\left[-b_{1}, b_{1}\right]$. Then, for $k \in \mathbb{R}$, and $\operatorname{sign} r_{-}(z(k))=$ $\operatorname{sign} k, R_{-}(z(k))=\frac{k-r_{-}(z(k))}{k+r_{-}(z(k))} e^{-2 i k \beta}+o\left(1 / k^{2}\right)$ and $R_{+}(z(k))=\frac{r_{-}(z(k))-k}{r_{-}(z(k))+k} e^{2 i r_{-}(z(k)) \beta}$ $+o\left(1 / k^{2}\right)$.

Proof. We give the proof for $R_{-}$. Using (17) and (18), for large $|k|, k \in \mathbb{R}$ we can write

$$
\phi_{+}(x, z(k))-\phi_{+, \beta}(x, z(k))=-R_{\beta}(z(k))\left(g_{1}-g_{2}+g_{3}\right)(x, k)
$$


where

$$
\begin{aligned}
& g_{1}(x, k)=\left(p \phi_{+, \beta}\right)(x, z(k)) \\
& g_{2}(x, k)=\left(p R_{\beta} p \phi_{+, \beta}\right)(x, z(k)), \\
& g_{3}(x, k)=\sum_{j=2}^{\infty}(-1)^{j}\left(\left(p R_{\beta}\right)^{j} p \phi_{+, \beta}\right)(x, z(k)) .
\end{aligned}
$$

Therefore,

$$
\begin{aligned}
& R_{-}(z(k))-\frac{k-r_{-}(z(k))}{k+r_{-}(z(k))} e^{-2 i k \beta} \\
& =\frac{-1}{W_{\beta}(z(k))} \frac{2 r_{-}(z(k))}{k+r_{-}(z(k))} e^{i \beta\left(r_{-}(z(k))-k\right)} \int \phi_{+, \beta}\left(x^{\prime}, z(k)\right)\left(g_{1}-g_{2}+g_{3}\right)\left(x^{\prime}\right) d x^{\prime} .
\end{aligned}
$$

Since $\left\|g_{3}(\cdot, z(k))\right\|=O\left(|k|^{-2}\right)$ for $k \in \mathbb{R}$, we need only concern ourselves with the contributions of $g_{1}$ and $g_{2}$.

We have

$$
\begin{aligned}
& \int \phi_{+, \beta}\left(x^{\prime}, z(k)\right) g_{1}\left(x^{\prime}, k\right) d x^{\prime} \\
& =H\left(\beta+b_{1}\right) \int_{-b_{1}}^{\beta} p\left(x^{\prime}\right) \frac{4 k^{2}}{\left(k+r_{-}(z(k))\right)^{2}} e^{2 i \beta\left(r_{-}(z(k))-k\right)} e^{-2 i r_{-}(z(k)) x^{\prime}} d x^{\prime} \\
& +H\left(b_{1}-\beta\right) \int_{\beta}^{b_{1}} p\left(x^{\prime}\right)\left(e^{-2 i k x^{\prime}}+\frac{2\left(k-r_{-}(z(k))\right)}{k+r_{-}(z(k))} e^{-2 i k \beta}\right. \\
& \left.\quad+\left(\frac{k-r_{-}(z(k))}{k+r_{-}(z(k))}\right)^{2} e^{-4 i \beta k} e^{2 i k x^{\prime}}\right) d x^{\prime}
\end{aligned}
$$

We will use the fact that $r_{-}(z(k))=k+O\left(|k|^{-1}\right)$. Using integration by parts in the first integral and in the first term of the second one, we obtain that (28) is equal to

$$
\begin{aligned}
& p(\beta)\left(\frac{4 k^{2} H\left(\beta+b_{1}\right)}{-2 i r_{-}(z(k))\left(k+r_{-}(z(k))\right)^{2}}+\frac{H\left(b_{1}-\beta\right)}{2 i k}\right) e^{-2 i \beta k} \\
& +\frac{H\left(\beta+b_{1}\right)}{2 i r_{-}(z(k))} \int_{-b_{1}}^{\beta} \frac{p^{\prime}\left(x^{\prime}\right) 4 k^{2}}{\left(k+r_{-}(z(k))\right)^{2}} e^{2 i \beta\left(r_{-}(z(k))-k\right) x^{\prime}} e^{-2 i r_{-}(z(k)) x^{\prime}} d x^{\prime} \\
& \quad+\frac{H\left(b_{1}-\beta\right)}{2 i k} \int_{\beta}^{b_{1}} p^{\prime}\left(x^{\prime}\right) e^{-2 i k x^{\prime}} d x^{\prime}+O\left(|k|^{-2}\right) .
\end{aligned}
$$

The first term is $O\left(|k|^{-2}\right)$ using again the fact that $r_{-}(z(k))=k+O\left(|k|^{-1}\right)$. If $h \in$ $L^{1}(\mathbb{R})$, then the Fourier transform $\hat{h}$ satisfies $\hat{h}(k)=o(1)$. Therefore, the two integrals in (29) contribute terms which are $o\left(|k|^{-1}\right)$, so that $\int \phi_{+, \beta}\left(x^{\prime}, z(k)\right) g_{1}\left(x^{\prime}, k\right)=$ $o\left(|k|^{-1}\right)$.

Next we consider $\int \phi_{+, \beta}\left(x^{\prime}, z(k)\right) g_{2}\left(x^{\prime}, k\right) d x^{\prime}$. Note that $\left\|g_{2}(\cdot, z(k))\right\|=O\left(|k|^{-1}\right)$ and $g_{2}$ has compact support, so that

$$
\int\left(\phi_{+, \beta}-e^{-i k x^{\prime}}\right)\left(x^{\prime}, z(k)\right) g_{2}\left(x^{\prime}, k\right) d x^{\prime}=O\left(|k|^{-2}\right) .
$$

Similarly, $\left\|g_{2}(x, k)-\left(p R_{\beta} p e^{-i k \cdot}\right)(x, z(k))\right\|_{L^{2}\left(\mathbb{R}_{x}\right)}=O\left(|k|^{-2}\right)$ so that we may further simplify our calculations. Moreover, using the explicit formula for the resolvent in 
terms of $\phi_{ \pm, \beta}$,

$$
\left\|\left(p R_{\beta} p(\cdot) e^{-i k \cdot}\right)(x, z(k))-\frac{1}{W_{\beta}(z(k))} p(x) \int e^{i k\left|x-x^{\prime}\right|} p\left(x^{\prime}\right) e^{-i k x^{\prime}} d x^{\prime}\right\|_{L^{2}\left(\mathbb{R}_{x}\right)}=O\left(|k|^{-2}\right) .
$$

Thus

$$
\begin{aligned}
& \int \phi_{+, \beta}\left(x^{\prime}, z(k)\right) g_{2}\left(x^{\prime}, k\right) d x^{\prime} \\
& \quad=\frac{1}{W_{\beta}(z(k))} \iint p\left(x^{\prime}\right) e^{-i\left(x^{\prime}+x^{\prime \prime}\right) k} e^{i k\left|x^{\prime \prime}-x^{\prime}\right|} p\left(x^{\prime \prime}\right) d x^{\prime} d x^{\prime \prime}+O\left(|k|^{-2}\right) .
\end{aligned}
$$

To show that (30) is $o\left(|k|^{-1}\right)$, we can integrate by parts in $x^{\prime \prime}$ when $x^{\prime \prime}<x^{\prime}$ and in $x^{\prime}$ when $x^{\prime \prime}>x^{\prime}$, thus finishing the proof of the lemma.

We shall use the previous lemma, Theorem 2.1, Proposition 4.1, and Proposition 4.4 to prove Theorem 1.2

Proof of Theorem 1.2. If $R_{-}(k)$ has no poles in $\operatorname{Im} k>0$ we shall apply Theorem 2.1 to the function $F(k)=R_{-}(z(k)) e^{2 i k \beta} / R_{-}(0)$. Note that for $k \in \mathbb{R}$, $F(k)=\left(V_{-}-V_{+}\right)\left(4 k^{2} R_{-}(0)\right)^{-1}+o\left(|k|^{-2}\right)$ as $|k| \rightarrow \infty$. Moreover, by Proposition 4.4. $h_{F}(\varphi)=2\left(b_{1}-\beta\right) \sin \varphi$. Then we may apply Theorem 2.1 to find that the number of zeros of $F(k)$ in the upper half plane with norm less than $r$ is given by $2\left(b_{1}-\beta\right)(\pi)^{-1} r+o(r)$. Then the first part of the theorem follows by applying Proposition 4.1 .

If $R_{-}$has any poles in the upper half-plane, it has only finitely many, and these may be handled as in the proof of Theorem 1.1. The second part of the theorem follows in an analogous way, using the estimates on $R_{+}$.

We now give the proof of the last of our principle forward results, Theorem 1.3 .

Proof of Theorem 1.3. By translation, we may assume that the convex hull of the support of $V^{\prime}$ is $\left[-b_{1}, b_{1}\right]$.

As before, use the coordinate $k=r_{+}(z)$ to describe points in the closure of the physical sheet of $\hat{Z}$. Consider the function $F(k)$ defined for $k \in \mathbb{C} \backslash$ $\left[-\sqrt{V_{-}-V_{+}}, \sqrt{V_{-}-V_{+}}\right]$by

$$
F(k)= \begin{cases}\frac{R_{-}(z(k))}{T_{-}(z(k))} & \text { if } \operatorname{Im} k \geq 0, k \notin\left[-\sqrt{V_{-}-V_{+}}, \sqrt{V_{-}-V_{+}}\right], \\ -\frac{R_{+}(z(-k))}{T_{-}(z(-k))} & \text { if } \operatorname{Im} k<0 .\end{cases}
$$

Note that $F(k)$ depends on the values of $R_{ \pm}, T_{-}$only on the closure of the physical sheet, although $F$ is defined on $\mathbb{C} \backslash\left[-\sqrt{V_{-}-V_{+}}, \sqrt{V_{-}-V_{+}}\right]$. We remark that $T_{-}(z(k))$ is nonzero in this region, and that if $T_{-}$has a pole, then $R_{ \pm}$has a pole of the same order at the same place. The function $R_{-}(z(k)) / T_{-}(z(k))$ is holomorphic on $\left\{k: \operatorname{Im} k \geq 0\right.$ and $\left.k \notin\left[-\epsilon-\sqrt{V_{-}-V_{+}}, \sqrt{V_{-}-V_{+}}+\epsilon\right]\right\}$, any $\epsilon>0$. A similar statement holds for $R_{+}(z(-k)) / T_{-}(z(-k))$ for $k$ in the closed lower half-plane. The relationships (5) and (8) combine to give, for $k \in \mathbb{R}, k \notin\left[-\sqrt{V_{-}-V_{+}}, \sqrt{V_{-}-V_{+}}\right]$,

$$
T_{-}(z(-k)) R_{+}(z(k))+R_{-}(z(-k)) T_{-}(z(k))=0 .
$$

This ensures that $F(k)$ is holomorphic on $\mathbb{C} \backslash\left[-\epsilon-\sqrt{V_{-}-V_{+}}, \sqrt{V_{-}-V_{+}}+\epsilon\right]$ for any $\epsilon>0$. From Propositions 4.3 and 4.4 , we know that

$$
h_{F}(\varphi)=2 b_{1} \sin (|\varphi|) \text {. }
$$


Thus applying Theorem 2.2. we obtain, using the notation of that theorem,

$$
n_{F, \sqrt{V_{-}-V_{+}}}(r)=\frac{4 b_{1}}{\pi} r+o(r) .
$$

It now remains to relate the zeros of $F$ to the poles of the resolvent in the desired region. The function $T_{-}$is nonzero, except, perhaps, at points which project to $V_{+}$. By Proposition 4.1 and (6), the zeros of $F$ in the upper half plane correspond, with multiplicity, to poles of the resolvent on the sheet $\left\{z \in \hat{Z}: \operatorname{Im} r_{+}<0, \operatorname{Im} r_{-}>\right.$ $0\}$. Similarly, using (11), the zeros of $F$ in the lower half-plane correspond, with multiplicity, to poles of the resolvent on the sheet $\left\{z \in \hat{Z}: \operatorname{Im} r_{+}>0, \operatorname{Im} r_{-}<0\right\}$. By Proposition 4.2, the zeros of $F(k)$ with $k \in \mathbb{R}$ coincide with poles at $z \in \hat{Z}$ with $\Pi(z) \in \mathbb{R}, \Pi(z)>V_{-}$, and $r_{+}(z)$ and $r_{-}(z)$ having opposite signs.

Finally, we include a proposition which we shall need for our inverse results.

Proposition 4.5. We have

$$
\sum_{\substack{z_{j} \in \mathcal{R} \\ r_{+}\left(z_{j}\right) \neq 0}} \frac{\left|\operatorname{Im}\left(r_{+}\left(z_{j}\right)\right)\right|}{\left|r_{+}\left(z_{j}\right)\right|^{2}}<\infty
$$

Proof. The proof follows from an application of Carleman's Theorem, using the variable $k=r_{+}(z)$, to, in turn, $R_{-}, R_{+}$, and $R_{-} R_{+}-T_{-} T_{+}$in the physical plane. We also use Propositions 4.2, 4.3, and 4.4.

\section{INVERSE RESULTS}

In this section we prove our inverse results. We must recover the reflection and transmission coefficients $R_{ \pm}, T_{ \pm}$on the boundary of the physical space. Because $V^{\prime}$ is compactly supported, this information is enough to recover the eigenvalues and the norming constants. Then, using results of 4], we recover the potential.

We will work with functions meromorphic in the plane whose zeros and poles are determined by $\mathcal{R}$. We can recover these functions, up to a finite number of unknown constants, by applying the Weierstrass factorization theorem. The difficulty is to find enough such functions to be able to recover $R_{ \pm}$and $T_{ \pm}$.

Lemma 5.1. For a real-valued steplike $V$, with $V^{\prime}$ compactly supported, $V_{+}, V_{-}$, and $\mathcal{R}$ determine

$$
R_{-}(z) R_{-}\left(w_{-}(z)\right), R_{+}(z) R_{+}\left(w_{+}(z)\right) \text {, and } T_{-}(z) / T_{-}\left(w_{+-}(z)\right) .
$$

Furthermore, $T_{-}(z) T_{-}\left(w_{-}(z)\right)$ and $T_{-}(z) T_{-}\left(w_{+}(z)\right)$ are determined up to constant real multiples.

Proof. Note that $R_{-}(z) R_{-}\left(w_{-}(z)\right)$ is a meromorphic function of $r_{+}(z)$. The poles of $R_{-}(z)$ are the same as the elements of $\mathcal{R}$, except that $R_{-}(z)$ is always regular at $\Pi^{-1}\left(V_{+}\right)$. The zeros of $R_{-}(z)$ are those $z^{\prime} \in \hat{Z}$ such that $w_{+}\left(z^{\prime}\right) \in \mathcal{R}, \Pi\left(z^{\prime}\right) \neq V_{+}$. Therefore, using Proposition 4.4 and the Weierstrass Factorization Theorem,

$$
R_{-}(z) R_{-}\left(w_{-}(z)\right)=\gamma_{1} e^{\delta_{1} r_{+}(z)} \prod_{z_{j} \in \mathcal{R}, \Pi\left(z_{j}\right) \neq V_{+}} \frac{r_{+}\left(z_{j}\right)+r_{+}(z)}{r_{+}\left(z_{j}\right)-r_{+}(z)},
$$

where $\gamma_{1}$ and $\delta_{1}$ are constants to be determined. The product converges using the fact that if $z_{j}$ is a resonance there is a resonance $z_{l}$ with $r_{+}\left(z_{l}\right)=-\overline{r_{+}}\left(z_{j}\right)$ and 
Proposition 4.5. But

$$
R_{-}(z) R_{-}\left(w_{-}(z)\right)=\frac{R_{-}(z)}{R_{+}(z)}\left(R_{-}(z) R_{+}(z)-T_{-}(z) T_{+}(z)\right)
$$

using (15), (6), (8) and (9). When $z$ lies on the physical sheet, using Propositions 4.3 and 4.4. this is a function of completely regular growth of type determined by the length of the convex hull of the support of $V^{\prime}$. By Theorem 1.1, this is determined by the resonances, so that the resonances determine $\delta_{1}$.

Fix $z^{\prime} \in \Pi^{-1}\left(V_{+}\right)$. Then $R_{-}\left(z^{\prime}\right)$ is 1 or -1 , by (6). It is -1 if $z^{\prime} \notin \mathcal{R}$ and 1 if $z^{\prime} \in \mathcal{R}$. This fixes the value of $R_{-}(z) R_{-}\left(w_{-}(z)\right)$ for $r_{+}(z)=0$, and thus determines $\gamma_{1}$.

A similar argument determines $R_{+}(z) R_{+}\left(w_{+}(z)\right)$ as a meromorphic function of $r_{-}(z)$.

Next we consider $T_{-}(z) T_{-}\left(w_{-}(z)\right)$ and $T_{-}(z) T_{-}\left(w_{+}(z)\right)$, which are meromorphic functions of $r_{+}(z)$ and $r_{-}(z)$, respectively. Note that $T_{-}(z)$ is nonzero, except, perhaps, if $z \in \Pi^{-1}\left(V_{+}\right)$. If $z^{\prime} \in \Pi^{-1}\left(V_{+}\right)$, then $T_{-}$has a zero of order 1 at $z^{\prime}$ if $z^{\prime} \notin \mathcal{R}$ and is nonzero at $z^{\prime}$ if $z^{\prime} \in \mathcal{R}$. Thus

$$
T_{-}(z) T_{-}\left(w_{-}(z)\right)=\gamma_{2} e^{\delta_{2} r_{+}(z)}\left(r_{+}(z)\right)^{\alpha_{+}} \prod_{z_{j} \in \mathcal{R}, \Pi\left(z_{j}\right) \neq V_{+}} \frac{1}{1-\frac{r_{+}(z)}{r_{+}\left(z_{j}\right)}},
$$

where $\alpha_{+}=1$ if $\Pi^{-1}\left(V_{+}\right) \cap \mathcal{R}=\emptyset$ and $\alpha_{+}=0$ if $\Pi^{-1}\left(V_{+}\right) \cap \mathcal{R} \neq \emptyset$. (Note that (11) ensures that at most one of the points which projects to $V_{+}$can be a resonance.) Again, the product converges by Proposition 4.5.

To see that $\delta_{2}$ is determined by $\mathcal{R}$, note that

$$
T_{-}(z) T_{-}\left(w_{-}(z)\right)=\frac{-r_{+}(z) T_{-}(z) T_{+}(z)}{r_{-}(z) R_{+}(z)} .
$$

Taking the reciprocal, we obtain a function which is, when $z$ is in the physical space, a function of completely regular growth as a function of $r_{+}(z)$. Just as for $R_{-}(z) R_{-}\left(w_{-}(z)\right)$, the type is determined by $\mathcal{R}$, and thus $\delta_{2}$ is determined. Since $T_{-}\left(w_{+}(z)\right) T_{-}\left(w_{+-}(z)\right)$ is the complex conjugate of $T_{-}(z) T_{-}\left(w_{-}(z)\right)$ when $V_{+}<\Pi(z)<V_{-}, \gamma_{2}$ is determined up to a real multiple.

A similar argument shows that $T_{-}(z) T_{-}\left(w_{+}(z)\right)$ is determined by $\mathcal{R}$, except for a real multiple.

Now

$$
\frac{T_{-}(z)}{T_{-}\left(w_{+-}(z)\right)}=\frac{T_{-}(z) T_{-}\left(w_{+}(z)\right)}{T_{-}\left(w_{+}(z)\right) T_{-}\left(w_{+-}(z)\right)}
$$

is therefore determined up to a constant, real, multiple. But, by Proposition 4.3 , $T_{-}(z) / T_{-}\left(w_{+-}(z)\right) \rightarrow 1$ when $z$ lies on the boundary of the physical sheet and $|z| \rightarrow \infty$. This, then, completely determines $T_{-}(z) / T_{-}\left(w_{+-}(z)\right)$.

The next lemma makes an assumption that $T_{-}(z) T_{-}\left(w_{-}(z)\right)$ is determined, which we have yet to prove. Proving this assumption is the sticking point in proving the inverse result in general. We are able to prove it in some special cases, and this is what enables us to prove the specialized inverse results.

Lemma 5.2. If $T_{-}(z) T_{-}\left(w_{-}(z)\right)$ is known, and $V \in L^{\infty}(\mathbb{R} ; \mathbb{R})$ is steplike, then $\mathcal{R}$, $V_{+}$, and $V_{-}$determine $R_{-}(z)$. 
Proof. Using (7),

$$
T_{-}(z) T_{-}\left(w_{-}(z)\right)=\frac{T_{-}(z) T_{-}\left(w_{+-}(z)\right)}{R_{-}\left(w_{+-}(z)\right)} .
$$

For the remainder of this proof, we assume that $z$ lies on the boundary of the physical space, with $\Pi(z)>V_{-}$. Then

$$
\bar{T}_{-}(z)=T_{-}\left(w_{+-}(z)\right), \bar{R}_{-}(z)=R_{-}\left(w_{+-}(z)\right) .
$$

Then, away from the zeros of $R_{-}\left(w_{+-}(z)\right), T_{-}(z) T_{-}\left(w_{-}(z)\right)$ determines the argument of $R_{-}\left(w_{+-}(z)\right)$, modulo $2 \pi$. Using (9) and (5),

$$
\frac{r_{-}(z)}{r_{+}(z)} \frac{T_{-}(z) T_{-}\left(w_{+-}(z)\right)}{R_{-}\left(w_{+-}(z)\right)}=\frac{1}{R_{-}\left(w_{+-}(z)\right)}-R_{-}(z) .
$$

Let $\rho=\rho(z)=\left|R_{-}(z)\right|$. Then

$$
f(z)=\left|\frac{r_{-}(z)}{r_{+}(z)} \frac{T_{-}(z) T_{-}\left(w_{+-}(z)\right)}{R_{-}\left(w_{+-}(z)\right)}\right|=\frac{1}{\rho}-\rho,
$$

where $f(z) \geq 0$ is known. Thus

$$
\rho=\frac{-f \pm \sqrt{f^{2}+4}}{2}
$$

If $R_{-}(z) \neq 0$ for all $z$ with $\Pi(z) \in\left[V_{-}, \infty\right)$, it is clear that we must take the "+" sign since $R_{-}(z) \rightarrow 0$ as $|z| \rightarrow \infty$ (recalling that $z$ lies on the boundary of the physical sheet, and using (9) and Proposition 4.3). On the other hand, if $R_{-}(z)$ does have such a zero, it is easy to see that we must take the "+" sign in the choice of $\rho$ to get $\rho=0$ at a pole of $f$.

Thus, for $z$ on the boundary of the physical space with $\Pi(z) \geq V_{-}$, we know the argument (modulo $2 \pi$ ) and norm of $R_{-}$, and thus know $R_{-}$there. Knowing $R_{-}$on an interval uniquely determines it on all of $\hat{Z}$.

Lemma 5.3. Suppose $V \in L^{\infty}(\mathbb{R} ; \mathbb{R})$ is steplike, $V^{\prime}$ has compact support, and $T_{-}(z) T_{-}\left(w_{-}(z)\right)$ is known. Then $\mathcal{R}, V_{+}$, and $V_{-}$determine $R_{+}(z)$ and $T_{-}(z)$.

Proof. By Lemma 5.1, we determine $R_{+}(z) R_{+}\left(w_{+}(z)\right)=R_{+}(z) / R_{+}\left(w_{+-}(z)\right)$ and $T_{-}(z) / T_{-}\left(w_{+-}(z)\right)$. By Lemma 5.2, $R_{-}(z)$ is determined, and by (5) and (9) we determine $T_{-}(z) T_{-}\left(w_{+-}(z)\right)$. By (10),$R_{+}(z) R_{+}\left(w_{+-}(z)\right)$ is determined. Therefore, $\left(T_{-}(z)\right)^{2}$ and $\left(R_{+}(z)\right)^{2}$ are fixed. But then $T_{-}(z)$ is determined by $T_{-}(z) \rightarrow 1$ as $|z| \rightarrow \infty$ with $z$ on the boundary of the physical sheet, and $R_{+}(z)$ is determined by $R_{+}\left(z_{1}\right)=-1$ when $\Pi\left(z_{1}\right)=V_{-}$.

By Lemma 5.2 and Lemma 5.3 and by applying results of [4, knowing $\mathcal{R}, V_{+}$, and $V_{-}$will determine a steplike $V$ (with $V^{\prime}$ compactly supported) provided that $T_{-}(z) T_{-}\left(w_{-}(z)\right)$ is fixed. Recall by Lemma 5.1 that $T_{-}(z) T_{-}\left(w_{-}(z)\right)$ is fixed by $\mathcal{R}$ up to a real, constant, multiple. We show how to fix this multiple in certain cases.

Lemma 5.4. If $\Pi^{-1}\left(V_{+}\right) \cap \mathcal{R} \neq \emptyset$, then, if $V \in L^{\infty}(\mathbb{R} ; \mathbb{R})$ is steplike with $V^{\prime}$ compactly supported, then $V_{+}, V_{-}$, and $\mathcal{R}$ determine $T_{-}(z) T_{-}\left(w_{-}(z)\right)$.

Proof. By Lemma 5.1, $T_{-}(z) T_{-}\left(w_{+}(z)\right)$ and $T_{-}(z) T_{-}\left(w_{-}(z)\right)$ are determined up to real constant multiples. We fix that multiple by determining what happens at one 
point. Fix $z_{0} \in \Pi^{-1}\left(V_{+}\right)$. If $z_{0} \in \mathcal{R}$, then $R_{-}\left(z_{0}\right)=1$ and $R_{-}\left(w_{-}\left(z_{0}\right)\right)=-1$. If $z_{0} \notin \mathcal{R}$, then $R_{-}\left(z_{0}\right)=-1$ and $R_{-}\left(w_{-}\left(z_{0}\right)\right)=1$. By (12),

$$
-\frac{r_{-}(z) T_{-}(z) T_{-}\left(w_{-}(z)\right)}{r_{+}(z)}=R_{-}(z)-R_{-}\left(w_{-}(z)\right) .
$$

We thus obtain

$$
\lim _{z \rightarrow z_{0}}-\frac{r_{-}(z) T_{-}(z) T_{-}\left(w_{-}(z)\right)}{r_{+}(z)}= \begin{cases}-2 & \text { if } z_{0} \notin \mathcal{R} \\ 2 & \text { if } z_{0} \in \mathcal{R} .\end{cases}
$$

Thus $T_{-}(z) T_{-}\left(w_{-}(z)\right)$ is fixed.

Lemma 5.5. Suppose $V$ is a real-valued steplike function, $V_{+}$and $V_{-}$are known, and it is known a priori that $V=p+V_{\beta}$ for some (unknown) $\beta \in \mathbb{R}$ and (unknown) $p \in C_{\text {comp }}^{0}(\mathbb{R})$ with $p^{\prime} \in L^{1}(\mathbb{R})$. Then $\mathcal{R}$ determines $T_{-}(z) T_{-}\left(w_{-}(z)\right)$.

Proof. Recall that

$$
T_{-}(z) T_{-}\left(w_{-}(z)\right)=-\frac{T_{-}(z) T_{-}\left(w_{+-}(z)\right)}{R_{-}\left(w_{+-}(z)\right)}
$$

and that by Lemma $5.1 T_{-}(z) T_{-}\left(w_{-}(z)\right)$ is determined up to a real constant multiple. Suppose that $z$ lies on the boundary of the physical space. By Lemma 4.2. $R_{-}\left(w_{+-}(z)\right)=\frac{V_{+}-V_{-}}{4\left(r_{+}(z)\right)^{2}} e^{2 i r_{+}(z) \beta}+o\left(\left(r_{+}(z)\right)^{-2}\right)$ as $|z| \rightarrow \infty$. Then, since $T_{-}(z) T_{-}\left(w_{+-}(z)\right) \rightarrow 1$,

$$
T_{-}(z) T_{-}\left(w_{+}(z)\right)=-\frac{4\left(r_{+}(z)\right)^{2}}{V_{+}-V_{-}} e^{-2 i r_{+}(z) \beta}+o\left(\left(r_{+}(z)\right)^{-2}\right)
$$

when $|z| \rightarrow \infty$ and $z$ is on the boundary of the physical sheet. This determines the (real) constant multiple, and thus $T_{-}(z) T_{-}\left(w_{-}(z)\right)$.

Lemma 5.6. Suppose that $V$ is a real-valued steplike function, and $V_{+}, V_{-}$, and $\mathcal{R}$ are known, with $\Pi^{-1}\left(V_{+}\right) \cap \mathcal{R}=\emptyset$. Let $z_{0} \in \hat{Z}$ such that $\Pi\left(z_{0}\right)=V_{+}$. Then if $T_{+}\left(z_{0}\right) / T_{+}\left(w_{-}\left(z_{0}\right)\right)>0, T_{-}(z) T_{-}\left(w_{-}(z)\right)$ is determined by $\mathcal{R}$.

We remark that by Lemma 5.1 and (5), $T_{+}\left(z_{0}\right) / T_{+}\left(w_{-}\left(z_{0}\right)\right)$ is determined by $\mathcal{R}$. This ratio must be real valued because for $z$ with $V_{+} \leq \Pi(z) \leq V_{-}, T_{+}(z)=$ $\bar{T}_{+}\left(w_{+}(z)\right)$ and $w_{+}\left(z_{0}\right)=z_{0}$. Checking this ratio in the simple case of a potential which takes on only three (distinct) values shows that this ratio can have either sign (see e.g. [18, Appendix 1]).

Proof. Since $z_{0}, w_{-}\left(z_{0}\right) \notin \mathcal{R}, R_{-}\left(z_{0}\right)=-1=R_{-}\left(w_{-}\left(z_{0}\right)\right)$. By (5) and (8), and using the fact that $w_{+}\left(z_{0}\right)=z_{0}$, we get

$$
R_{+}\left(z_{0}\right)=-R_{-}\left(w_{-}\left(z_{0}\right)\right) \frac{T_{+}\left(z_{0}\right)}{T_{+}\left(w_{-}\left(z_{0}\right)\right)} .
$$

Our assumption on the sign of $T_{+}\left(z_{0}\right) / T_{+}\left(w_{-}\left(z_{0}\right)\right)$ shows that $R_{+}\left(z_{0}\right)>0$, and thus $R_{+}\left(w_{-}\left(z_{0}\right)\right)>0$ as well.

Let $z_{1} \in \hat{Z}$ have $\Pi\left(z_{1}\right)=V_{-}$. We have already noted that since $z_{1} \notin \mathcal{R}$, $R_{+}\left(z_{1}\right)=-1$. Because $R_{+}$is continuous at all points $z$ with $\Pi(z) \in\left[V_{+}, V_{-}\right]$and is nonzero is this range, there is some point $z^{\prime}$ with $\Pi\left(z^{\prime}\right) \in\left(V_{+}, V_{-}\right)$and $R_{+}\left(z^{\prime}\right)$ pure imaginary. At this point, $R_{+}\left(w_{+}\left(z^{\prime}\right)\right)=-R_{+}\left(z^{\prime}\right)$ and $r_{+}\left(z^{\prime}\right) T_{+}\left(z^{\prime}\right) T_{+}\left(w_{+}\left(z^{\prime}\right)\right)=$ $-2 r_{-}\left(z^{\prime}\right) R_{+}\left(z^{\prime}\right)$ by (13). That is, $\left|r_{+}(z) T_{+}(z) T_{+}\left(w_{+}(z)\right) / r_{-}(z) R_{+}(z)\right|$ is a maximum at $z=z^{\prime}$ for $\Pi(z) \in\left[V_{+}, V_{-}\right]$. Note that for $\Pi(z) \in\left[V_{+}, V_{-}\right],\left|R_{+}(z)\right|=$ 
$\left(R_{+}(z) R_{+}\left(w_{+}(z)\right)\right)^{1 / 2}$ is known. Thus the norm of $r_{+}\left(z^{\prime}\right) T_{+}\left(z^{\prime}\right) T_{+}\left(w_{+}\left(z^{\prime}\right)\right) / r_{-}\left(z^{\prime}\right)$ $=-2 R_{+}\left(z^{\prime}\right)$ is known. Since $T_{+}(z) T_{+}\left(w_{+}(z)\right)$ was already determined up to a real constant multiple and $T_{+}\left(z_{0}\right) T_{+}\left(w_{+}\left(z_{0}\right)\right)>0$, it is completely determined. Thus, by (5), $T_{-}(z) T_{-}\left(w_{+}(z)\right)$ is determined, and by Lemma 5.1, $T_{-}(z) T_{-}\left(w_{-}(z)\right)$ is fixed.

We summarize our inverse results in the following theorem.

Theorem 5.1. Knowledge of $V_{+}, V_{-}$, and $\mathcal{R}$ uniquely determines, up to translation, a real steplike potential $V$ with $V^{\prime}$ compactly supported provided at least one of the following criteria is met:

- $\Pi^{-1}\left(V_{+}\right) \cap \mathcal{R} \neq \emptyset$.

- It is known a priori that $V=V_{\beta}+p$ for some (unknown) $\beta \in \mathbb{R}$ and (unknown) $p \in C_{\text {comp }}^{0}(\mathbb{R})$ with $p^{\prime} \in L^{1}(\mathbb{R})$.

- For $z_{0} \in \Pi^{-1}\left(V_{+}\right), T_{+}\left(z_{0}\right) / T_{+}\left(w_{-}\left(z_{0}\right)\right)>0$.

\section{REFERENCES}

[1] B.M. Brown, I. Knowles, and R. Weikard, On the inverse resonance problem, J. London Math. Soc. (2) 68 (2003), no. 2, 383-401. MR1994689 (2004b:34026)

[2] T. Christiansen, Some upper bounds on the number of resonances for manifolds with infinite cylindrical ends, Annales Henri Poincaré 3 No. 5 (2002), 895-920. MR1937607|(2003i:58054)

[3] T. Christiansen, Asymptotics for a resonance-counting function for potential scattering on cylinders, J. Funct. Anal. 216 (2004), no. 1, 172-190. MR2091360

[4] A. Cohen and T. Kappeler, Scattering and inverse scattering for steplike potentials in the Schrödinger equation, Indiana Univ. Math. J. 34 (1985), no. 1, 127-180. MR0773398 (86k:34017)

[5] R. Froese, Asymptotic distribution of resonances in one dimension, J. Differential Equations 137 (1997), no. 2, 251-272. MR.1456597 (98f:81339)

[6] R. Froese, Upper bounds for the resonance counting function of Schrödinger operators in odd dimensions, Canad. J. Math. 50 (1998), no. 3, 538-546. MR1629819 (99f:35150)

[7] L. Guillopé, Théorie spectrale de quelques variétés à bouts, Ann. Scient. Ec. Norm. Sup. 22, 4, (1989), 137-160. MR0985859 (90g:58136)

[8] E. Korotyaev, Inverse resonance scattering on the real line, preprint.

[9] E. Korotyaev, Inverse resonance scattering on the half line, Asymptot. Anal. 37 (2004), no. 3-4, 215-226. MR2047740 (2005a:34010)

[10] B. Ja. Levin, Distribution of zeros of entire functions, American Mathematical Society, Providence, R.I., 1964, viii+493 pp. MR0156975 (28:217)

[11] R.B. Melrose, The Atiyah-Patodi-Singer Index Theorem, A.K. Peters, Wellesley, MA, 1993. MR1348401 (96g:58180)

[12] L. Parnovski, Spectral asymptotics of the Laplace operator on surfaces with cusps, Math. Ann. 303 (1995), 281-296. MR.1348800 (97a:11080)

[13] T. Regge, Analytic properties of the scattering matrix, Nuovo Cimento 8 (5), (1958), 671-679. MR0095702 (20:2203)

[14] B. Simon, Resonances in one dimension and Fredholm determinants, J. Funct. Anal. 178 (2000), no. 2, 396-420. MR.1802901 (2001j:34031)

[15] J. Sjöstrand, Resonances for bottles and trace formulae, Math. Nachr. 221, (2001), 95-149. MR:1806367 (2001k:58063)

[16] G. Vodev, Asymptotics on the number of scattering poles for degenerate perturbations of the Laplacian, J. Funct. Anal. 138 (1996), 295-310. MR1395960 (97g:35125)

[17] G. Vodev, Resonances in the Euclidean scattering, Cubo Matemática Educacional 3 no. 1 (2001), 317-360.

[18] R. Weder, Spectral and scattering theory for wave propagation in perturbed stratified media, Springer-Verlag, New York, 1991. MR 1082152 (91j:35198)

[19] M. Zworski, Distribution of poles for scattering on the real line, J. Funct. Anal. 73 (2) (1987), 277-296. MR0899652(88h:81223) 
[20] M. Zworski, Counting scattering poles. In: Spectral and scattering theory (Sanda, 1992), 301-331, Lecture Notes in Pure and Appl. Math., 161, Dekker, New York, 1994. MR1291649 (95i:35210)

[21] M. Zworski, Resonances in physics and geometry. Notices Amer. Math. Soc. 46 (1999), 319328. MR.1668841 (2000d:58051)

[22] M. Zworski, A remark on isopolar potentials, SIAM J. Math. Analysis, 82 (6) (2001), 18231826. MR1856251

Department of Mathematics, University of Missouri, Columbia, Missouri 65211

E-mail address: tjc@math.missouri.edu 